
Annual Technical Progress Report to the

Department of Energy

Atmospheric Radiation and Measurement Program (ARM)

for Year 1:

1 Dec 91 - 30 Nov 92

\title{
Parameterization of GCM Subgrid Nonprecipitating Cumulus and Stratocumulus Clouds Using Stochastic/ Phenomenological Methods
}

Grant Number: DE-FG02-92ER61361

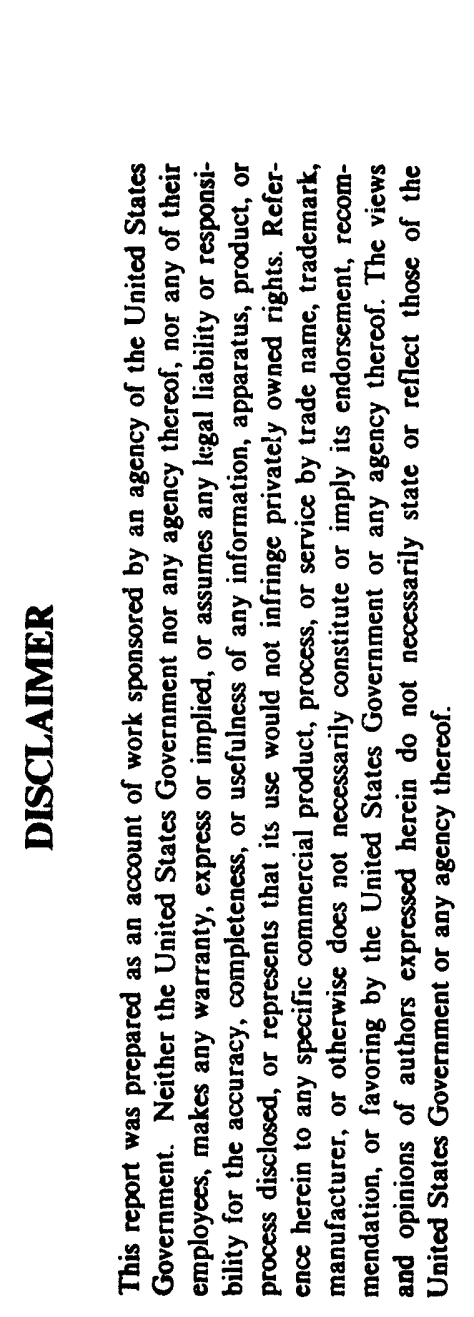

\author{
P.I.: Prof. Roland B. Stull
}

Boundary Layer Research Team

Department of Atmospheric and Oceanic Sciences

University of Wisconsin - Madison

1225 W. Dayton St.

Madison, WI 53706

Report Date: 26 Aug 92 


\section{Summary of Overall Progress}

We have had a very successful research year in the development of new theories, analysis of field data, and formulation of preliminary parameterizations of cloud cover for GCMs. Details follow.

\section{a. Personnel}

PI: Prof. Roland Stull

Ph.D. Student: Qing Zhang

MS. Student: Joe Farrenkopf

MS. Student: Kelly McNerney

Undergrad. Computer Sci. Major: Rudy Moore

Undergrad. Computer Sci. Major: Rizwan Qureshi

\section{b. Activities}

Shortly after the grant was awarded, Stull developed a theory for the fraction of near-surface air likely to form "forced" and "active" cumulus clouds. This stochastic method is based on a 2-D frequency distribution of the occurrence of various buoyancy and condensation levels in air near the surface, and the relationship of this distribution to the mean temperature profile. An alternative form of this theory, developed by Stull after a stimulating visit by Dave Randall to our department, utilizes a 2-D distribution of convective available potential energies (CAPE) instead of virtual potential temperatures.

A workstation was purchased, along with two digital audio tape (DAT) drives to archive the massive amounts of turbulence data needed to do this study. The second DAT drive was attached to one of our existing workstations.

Existing aircraft data from the Hydrologic-Atmospheric Pilot Experiment (HAPEX) field experiment from France was used to begin testing this theory. As a first step, Ms. McNerney examined the microfilm summaries of the aircraft data from NCAR, and determined the starting and ending times of the needed flight legs. Then she modified some existing programs from NCAR and our own group to unpack the aircraft data and store it in a more-easily accessible form on DAT tapes connected to our workstations here at Wisconsin. 
Ms. Zhang, who recently passed her Ph.D. qualifying exam, is conducting some of this DOE-ARM research as the basis of her dissertation. She began by calibrating the aircraft data and computing preliminary 2-D frequency distributions for both virtual potential temperature and CAPE, as shown in the results section below. She also analyzed the sounding data from aircraft, and made the preliminary diagnoses of cloud cover. Ms. Zhang is also supervising IMcNerney.

Mr. Farrenkopf, who is finishing is MS thesis in a different topic, joined the ARM project this summer to start developing a 1-D column parameterization of boundary-layer clouds for use in a GCM. His MS work had included deveiopment of a boundary-layer forecast model, which provided him with some experience that can be applied to GCMs.

The two computer science majors are employed as undergraduate hourly workers to develop special graphic algorithms to help display the massive announts of aircraft turbulence data. Their computer programs were used by Ms. Zhang to examine the raw data for errors, and to perform some of the initial comparisons of frequency distribution to mean temperature profile (e.g., Fig 2).

Ms. Zhang's summary of results to date have been accepted for presentation as a poster at the upcoming American Meteorological Society turbulence conference in Portland. A copy of the preprint paper is attached.

Meanwhile, as an independent research direction, Stull developed a convective drag theory to relate surface fluxes to mixed-layer characteristics. This has the potential to allow GCMs with poor (or no) vertical resolution in the surface layer to diagnose fluxes under conditions of free convection. He tosted this theory against existing published data sets from the Koorin field experiment in Australia, and the BLX83 field program in Oklahoma. These results were written as a paper submitted to the J. of Atmos. Sci., which is also attached. The results have also been accepted for presentation to the AMS turbulence conference, and the preprint paper is attached.

Because the depth of the mixed layer is a critical parameter needed in many other theories, Stull began developing a new theory to explain the levelness of the mixed-layer top over irregular topography. This has obvious application to GCMs, where topography is an important aspect of the model. Although this research is still in the very preliminary stages, a paper about it has been accepted for presentation at the AMS turbulence conference. A copy is attached. 


\section{c. Results to date}

McNerney has unpacked the data from Flight 6 (21 May 86) and Flight 1 (9 May 86) of HAPEX. From this data, there were 18 flight legs that were flown near the surface. These legs were flown over a three types of land-use conditions: flat evergreen forest on sandy soil, mixed crops on gentle hills, and farms and pastures on steeper hills. We are grouping similar flight legs in order to help recognize repeated patterns in the results.

We currently have 6 frequency distributions for each land-use type. Fig 1 shows a single sample from each type from the HAPEX data set. These distributions show similarities and differences between difference land uses, which we will try to explain and parameterize.

Fig 2a shows the superposition of a vertical temperature profile with one of the 2-D frequency distributions. According to the theory, those data points from the near-surface flight leg plotted to the right of the sounding curve are likely to form clouds. The fraction of points satisfying this condition gives the fraction of sky covered by clouds. For Fig $2 a$, the theoretical fractional cloud cover of $37 \%$ is slightly below the observed cloud cover of 40 to $50 \%$. The subset of clouds that are "active" according to our theory is $33 \%$, which also is on the low side of the observed range of $30-50 \%$.

A similar analysis can be made for CAPE, as is shown in Fig $2 b$ for the same case. This method has the advantage of including the vertically integrated effects of buoyancy, vertical inertia, and allows easier incorporation of other cloudformation mechanisms such as wind-shear-generated turbulence. This method yields a diagnosed active cloud cover of $57 \%$, and total cloud cover of $65 \%$. These values are slightly larger than observed. It appears that a parameterization based on a combination of the CAPE and temperature methods might yield the best diagnoses of cloud cover.

Of the 8 diagnoses of cloud cover made so far, the CAPE and temperature methods correctly bracketed 7 of the corresponding observations. For the remaining 10 flight legs, inadequate sounding data precluded a test of the theory. 

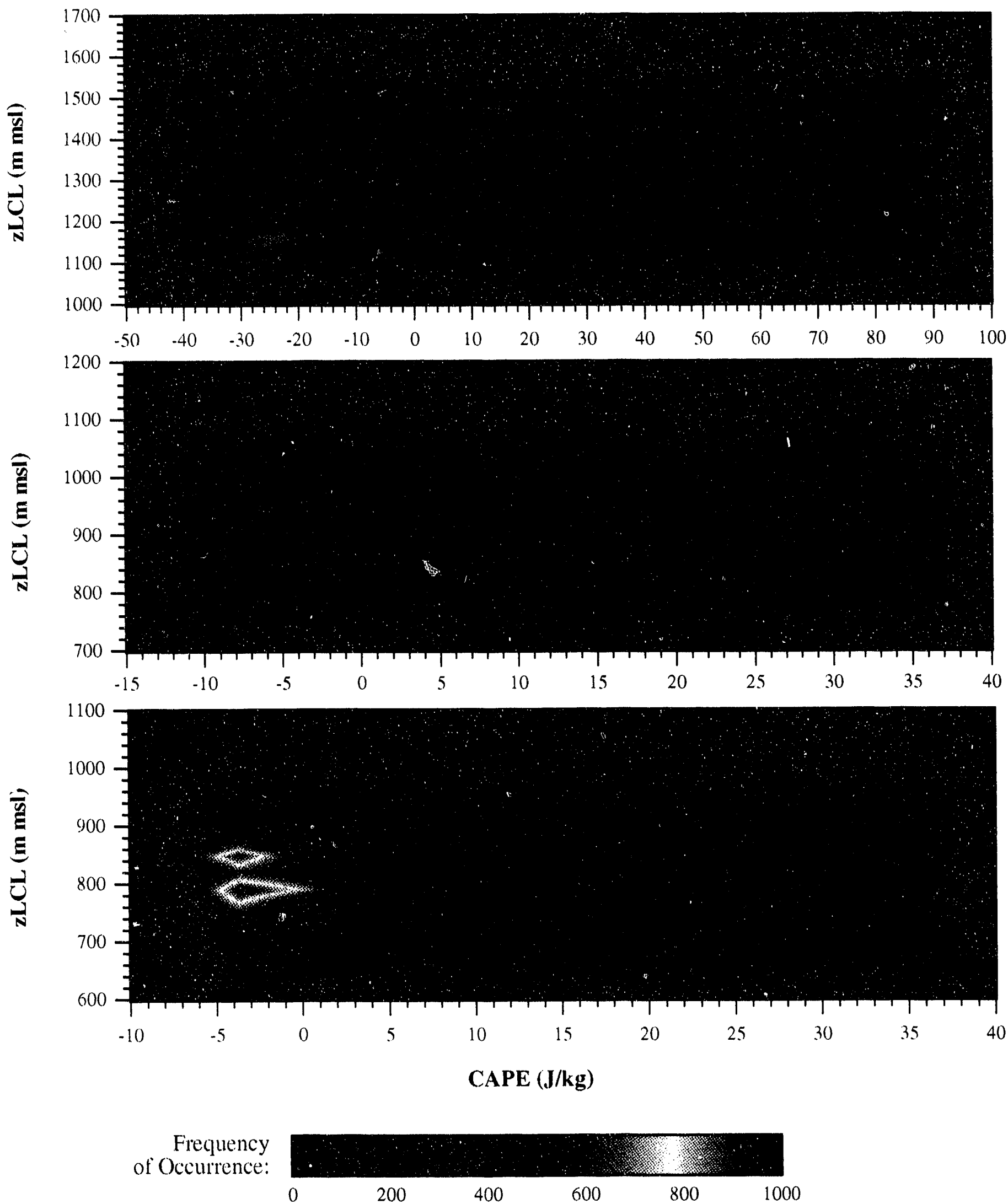

Fig. 1. Aircraft observations in the surface layer of frequency of occurrence (colors) of air with various lifting-condensation-level heights ( $\mathrm{ZLCL}$ ) and relative convective available potential energies (CAPE), evaluated at the LCLs. Land use: (a) flat evergreen forest; (b) small hills with mixed agriculture; and (C) larger hills with mixed agriculture and pasture. 
R. Stull, Univ. of Wisconsin

GCM Subgrid Cumulus
Progress Report to DOE-ARM

Year 1 page 6
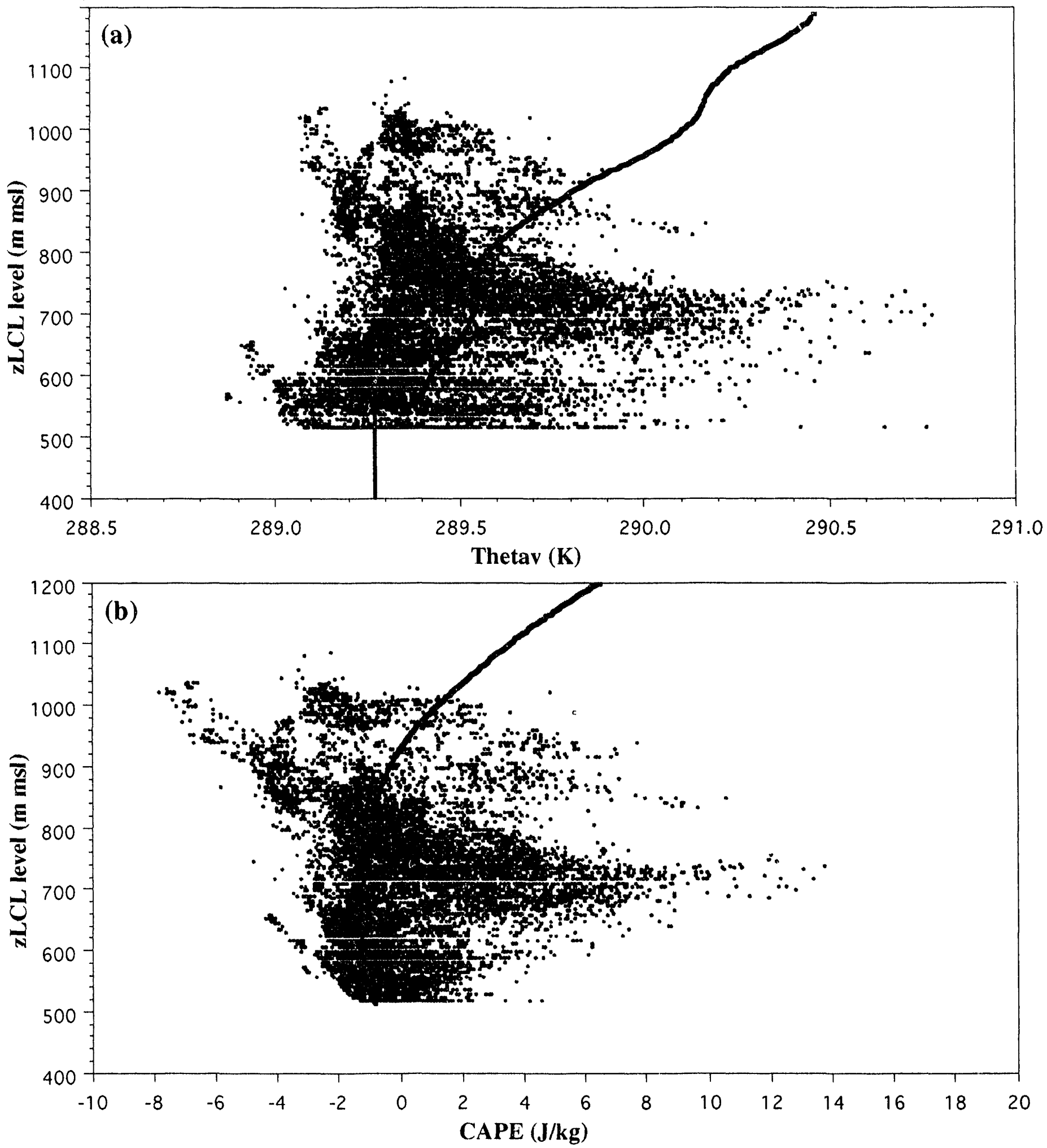

Fig 2. (a) Aircraft data measured every $1 / 20$ s near the surface (cluster of points) of height of the lifting condensation level (ordinate) vs virtual potential temperature (abscissa). Superimposed is average sounding (solid line) of height (ordinate) vs virtual potential temperature (abscissa). (b) Same, but with CAPE along abscissa. 


\section{d. Relationship to general goals.}

Each flight leg is over a distance of about $50 \mathrm{~km}$, which corresponds to less than a single grid cell in a typical GCM. Thus, our data represents subgrid information. By parameterizing this distribution as a function of known surface conditions and resolved GCM mean variables, we can use it to forecast the amount of subgrid clouds. By incorporating parameters into the modeled frequency distribution, and calibrating those parameters based on aircraft data from various ARM-CART sites around the world, we should be able to develop a parameterization with wide utility.

\section{Unusual Developments}

The addition of Kelly McNerney and Joe Farrenkopf to this project during the middle of the grant year was unanticipated, but fortuitous because they are both talented individuals with experience and enthusiasm that could greatly benefit the research project. In fact, McNerney was awarded an American Meteorological Society fellowship, starting this fall. However, the original budget for the first year including funding for only one graduate student, Qing Zhang. Thus, the budget was strained this first year. As a result, Stull did not charge any of his time against the grant, in order to save money to pay for the graduate students. Because the second year budget includes funds for two graduate students (Zhang and Farrenkopf), and McNerney requires only partial funding because of her fellowship, we expect the present funding stress to be relieved.

Additional field experiment data was acquired from NCAR on Exabyte tapes, rather than conventional real-to-real data tapes. We specifically asked for the Exabyte tapes because of the promise of greater storage capabilities and longer shelf life of the data. However, thest have proved difficult to read and unpack. McNerney is making progress, thanks to unpacking programs provided by other scientists from NCAR. If worse comes to worse, we can always reorder the data from NCAR on traditional computer tapes. We expect to have this problem solved in about a month. 


\section{3: Plans for the remainder of year 1}

During the next three months Zhang, McNerney and Stull will analyze data from about two more days from HAPEX. This work should proceed much faster, as all of the necessary techniques and computer programs have been developed.

Farrenkopf will begin to modify an existing column model for turbulence to add forecast capability for moisture, and to compute condensation levels and frequency distributions.

Stull revise the convective drag paper, and resubmit it to the J. of Atmos. Sci.

Stull, Zhang, Farrenkopf, and McNerney will attend the AMS conference in late September, and Stull will attend the ARM workshop later this fall.

\section{Summary of Work to be Preformed During Year 2}

Based on the HAPEX data as a starting point, Zhang and Stull will develop parameterizations for the 2-D frequency distributions. This will require some preliminary work to calculate the boundary layer scaling parameters from the field experiment data.

Farrenkopf will incorporate the resulting parameterization into his 1-D column model of the boundary layer, and will make some sample forecasts to compare with observations here in Madison.

ARM-CART data will be utilized as soon as it is available. This includes data from aircraft flight legs, soundings, surface turbulence and radiation budgets, and observations of cloud cover, type, and depth. Additional data from other existing data sets, such as FIFE and/or STORM-FEST, will be acquired and analyzed. An additional workstation will be acquired to aid the processing of all this data. Results from these data will be used to refine the parameterizations.

A paper about parameterization of cloud cover and type will be written and submitted for publication in a journal.

Work will be initiated to study the cloud size distributions and dynamical category, and to parameterize those in terms of GCM resolved variables. 
Theories concerning convective drag theory will be refined and tested against other data sets. A paper about mixed-layer levelness will be written for publication.

\section{List of Publications Resulting from this Research}

Stull, R.B., 1992: A convective drag theory for surface fluxes. (submitted to J. Atmos. Sci., Apr 92)

Stull, R.B., 1992: A theory for mixed-layer top levelness over irregular topography. Preprint Volume of the American Meteorological Society's 10th Symposium on Turbulence and Diffusion, 29 Sep - 2 Oct 1992, Portland, OR. American Meteorological Society, 45 Beacon St. Boston, MA 02108. (in press).

Stull, R.B., 1992: A convective drag theory for surface fluxes. Preprint Volume of the American Meteorological Society's 10th Symposium on Turbulence and Diffusion, 29 Sep - 2 Oct 1992, Portlard, OR. American Meteorological Society, 45 Beacon St. Boston, MA 02108. (in press).

Zhang, Q., 1992: Diagnostic determination of boundary-layer cumulus clouds using HAPEX aircraft data. Preprint Volume of the American Meteorological Society's 10th Symposium on Turbulence and Diffusion, 29 Sep - 2 Oct 1992, Portland, OR. American Meteorological Society, 45 Beacon St. Boston, MA 02108. (in press).

Copies of these publications are attached.

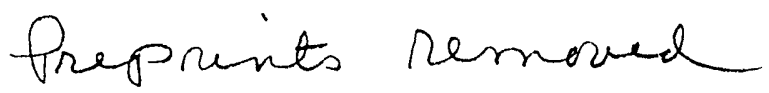

\section{Incremental Budget Request for Year 2 Funding}

The funds for year 1 will be totally spent by the end of the first budget year. A copy of the budget for year 2 , which has not changed from our original request, is on the next page.

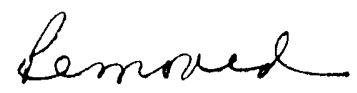



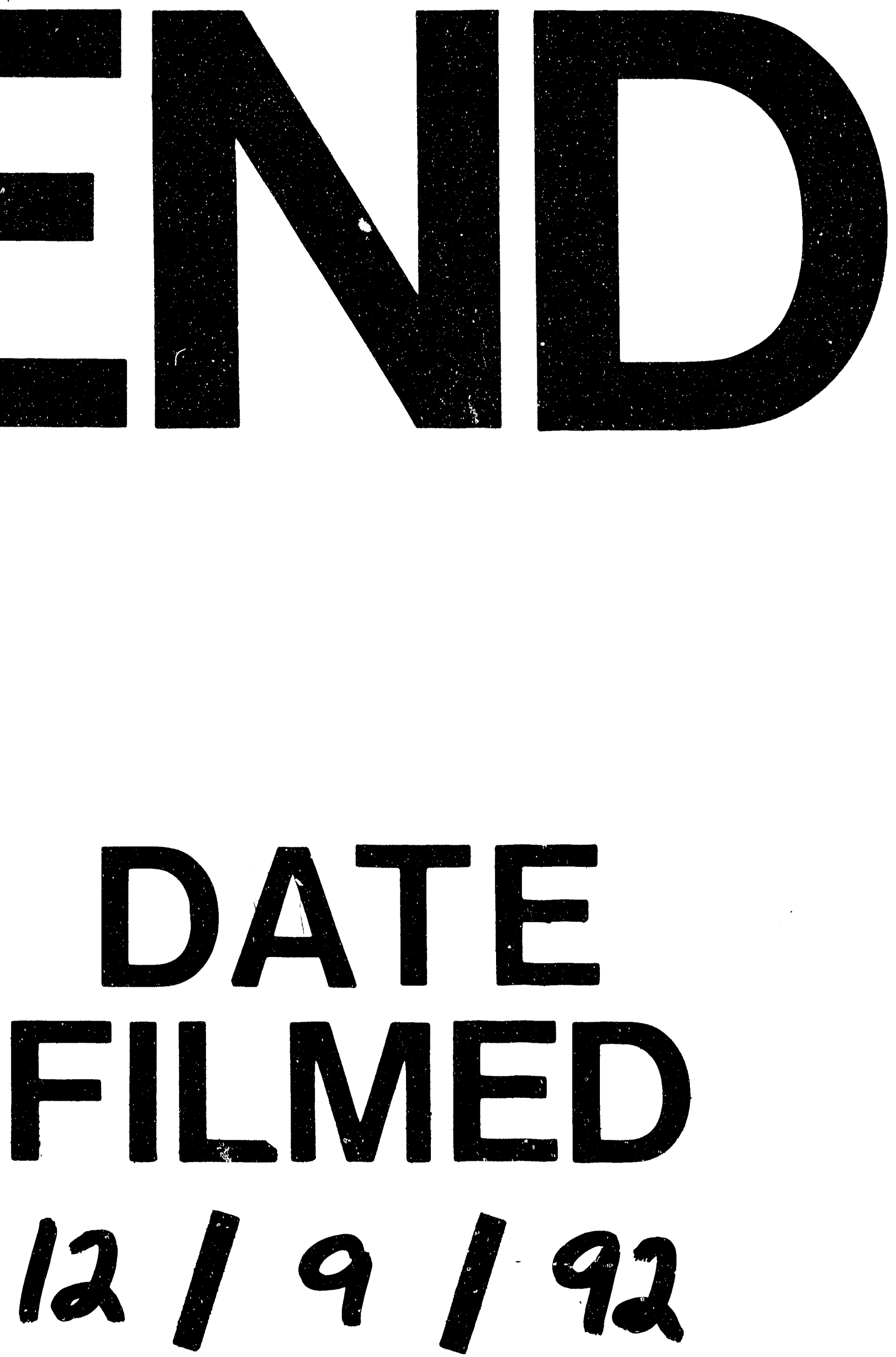
